\title{
三维氮掺杂碳纳米带的制备及其在锂硫电池中的应用
}

\author{
李宛飞 ${ }^{a}$ 马倩 ${ }^{a}$ 郑召召 ${ }^{a}$ 张跃钢*,a,b \\ ( ${ }^{a}$ 中国科学院苏州纳米技术与纳米仿生研究所 国际实验室 苏州 215123) \\ ( ${ }^{b}$ 清华大学物理系 北京 100084)
}

\begin{abstract}
摘要 锂硫电池凭借其高的理论能量密度 $\left(2600 \mathrm{~W} \cdot \mathrm{h} \cdot \mathrm{kg}^{-1}\right)$ 、丰富廉价的材料来源、且对环境友好等优势，而引起了人 们的广泛关注. 然而, 锂硫电池活性物质导电性差、多硫化物易溶于有机电解液等问题所导致的硫正极倍率性能和循 环稳定性差，仍然是困扰锂硫电池发展的挑战性难题. 我们设计并以廉价易得的小分子化合物对苯二酚和甲醛为原料, 通过缩聚反应、与氧化石墨烯原位复合、高温氮化制备了一类新型氮掺杂的碳纳米带固硫载体材料(NCNB-NG). 通过 NCNB-NG 复合纳米硫进一步得到的碳-硫复合正极材料(S@NCNB-NG)表现出更优异的倍率性能和循环稳定性, 这主 要得益于该碳质载体独特的微结构以及改善的导电性.
\end{abstract}

关键词 锂硫电池; 碳; 氮掺杂; 纳米带; 三维

\section{Preparation of Three-dimensional Nitrogen-doped Carbon Nanoribbon and Application in Lithium/Sulfur Batteries}

\author{
Li, Wanfei $^{a} \quad$ Ma, Qian $^{a} \quad$ Zheng, Zhaozhao $^{a} \quad$ Zhang, Yuegang*,a,b \\ ( ${ }^{a}$-Lab, Suzhou Institute of Nano-Tech and Nano-bionics, Chinese Academy of Sciences, Suzhou, Jiangsu, 215123) \\ ( ${ }^{b}$ Department of Physics, Tsinghua University, Beijing, 100084)
}

\begin{abstract}
Lithium/sulfur (Li-S) batteries have recently attracted intensive research interests due to their high theoretical specific energy of $2600 \mathrm{~W} \cdot \mathrm{h} \cdot \mathrm{kg}^{-1}$. However, the poor electronic conductivity of sulfur and the high solubility of polysulfides in organic electrolytes lead to poor cycling stability and rate capability. Herein, we report a three-dimensional (3D) nanocomposite network made from nitrogen-doped carbon nanoribbon (NCNB) and nitrogen-doped graphene (NG), which has a high electronic conductivity and can serve as a conductive matrix and a sulfur immobilizer for the sulfur cathode. The NCNB is prepared by thermal nitridation of a unique 3D phenolic resin (PHF) isolated from the polycondensation reaction of 1,4-hydroquinone and formaldehyde. The N content of NCNB-NG can reach as high as $9.7 \mathrm{wt} \%$. Although three types of $\mathrm{N}$ bonding geometries, including pyridinic N, pyrrolic N, and graphitic N, are identified in the NCNB-NG composites, we found the pyridinic $\mathrm{N}$ is dominant, which facilitates the trapping of intermediate lithium polysulfides. The sulfur was loaded on NCNB-NG by using a $\mathrm{Na}_{2} \mathrm{~S}_{2} \mathrm{O}_{3}$ solution as sulfur source. The scanning electron microscope (SEM) images show that almost no large S particle can be observed in the as-prepared S@NCNB-NG nanocomposites, suggesting a uniform coating of $\mathrm{S}$ on the NCNB-NG networks. The transmission electron microscopic (TEM) images and the elemental mapping by Energy-Dispersive X-ray (EDX) analysis also show that nano-sized S particles are uniformly distributed on the NCNB-NG matrix. The as-obtained S@NCNB-NG cathode can deliver a high specific capacity of $1338 \mathrm{~mA} \cdot \mathrm{h} \cdot \mathrm{g}^{-1}$ at $0.05 \mathrm{C}$ with about $80 \%$ $\mathrm{S}$ utilization. It also exhibits excellent rate capability and good cycle stability with a retained specific capacity of $556 \mathrm{~mA} \bullet$ • $\mathrm{g}^{-1}$ after 300th cycles. These performances are much higher than the control samples using the S@NCNB and the S@PHF nanocomposites as cathodes. The improved performance can be attributed to the unique microstructure and the improved electronic conductivity of the NCNB-NG matrix.
\end{abstract}

Keywords lithium/sulfur battery; carbon; N-doped; nanoribbon; three-dimensional

\section{1 引言}

近些年来, 锂硫电池由于具有高理论能量密度和硫 资源丰富、价格低廉、环境友好等优势, 而在电化学储 能领域受到广泛关注 ${ }^{[1 \sim 4]}$. 然而到目前为止, 糟糕的硫 正极循环稳定性仍然是限制其产业化应用的重要瓶颈
和亟待解决的难题 ${ }^{[57]}$. 目前, 硫正极结构 ${ }^{[8 \sim 17]}$ 、电解液 优化 ${ }^{[18 \sim 20]}$ 和隔膜修饰技术 ${ }^{[21 ~ 27]}$ 等策略已经被用来提升 硫正极的循环稳定性能. 其中以纳米碳作为硫的载体以 及导电骨架的碳硫复合正极材料的研究成果尤为显著 而备受关注. 例如，凭借石墨烯和碳纳米管高的比表面

*E-mail: ygzhang2012@sinano.ac.cn; Tel: 0512-62872772

Received August 25, 2016; published December 5, 2016.

Supporting information for this article is available free of charge via the Internet at http://sioc-journal.cn.

Project supported by the Natural Science Foundation of China (Nos. 21433013, 51402345, 21403287), and the Suzhou Science and Technology Development Program (Nos. ZXG2013002, SYG201532).

项目受国家自然科学基金(Nos. 21433013, 51402345, 21403287)和苏州市科技计划项目(Nos. ZXG2013002, SYG201532)资助. 
积、优异的导电性以及其氮或/和氧功能化后的强化学健 合固硫能力, 硫-石墨烯复合物 ${ }^{[10,11]}$ 和硫-碳纳米管复合 物 ${ }^{[12]}$ 展现出了高性能, 并已取得了重大的研究进展. 但 从应用的角度, 石墨烯和碳纳米管或者功能化石墨烯和 碳纳米管存在成本比较高, 且其制备工艺要么复杂、要 么用到大量环境不友好的试剂、要么高能耗等问 题 ${ }^{[28 ~ 37]}$. 因此, 探索和发展合成方法简单、环保、成本 低廉、易规模化生产的高效功能化硫载体碳质材料具有 重要的意义.

2009 年, 加拿大的 Nazar 研究组 ${ }^{[8]}$ 首次利用廉价的 工业聚合物原料聚甲基丙烯酸甲酯(PMMA)发展了一种 新型硫载体介孔碳质材料(CMK-3), 并将其成功应用于 复合纳米硫构建硫正极, 通过把硫进行纳米级分散并与 纳米导电碳质材料复合, 实现了活性物质硫的利用率和 电池循环稳定性的大幅提高. 随后, Liu 等 ${ }^{[38]}$ 利用另一 个工业上常用的廉价聚合物酚醛树脂作为碳源，制备了 一种结构独特的硅一碳纳米复合物, 并将其用于超级电 容器, 表现出了优异的循环稳定性. 从此, 基于聚合物 作为碳源制备碳质载体策略迅速在电化学储能领域引 起了人们的注意, 并得到了快速的发展. 但到目前为止, 基于绿色廉价的酚醛树脂发展碳材料并考察其应用于 锂硫电池的工作还非常少.

前期工作中, 我们研究小组将氧化石墨烯 $(\mathrm{GO})$ 以 及氧化石墨烯改进后生成的氮掺杂石墨烯(NG)通过复 合纳米硫制备出了性能优异的硫正极材料 ${ }^{[15,16]}$. Guo 研 究组 ${ }^{[39}$ 将嗍掺杂的多孔碳(BPC)作为硫的碳载体材料制 备的硫正极材料(S/BPC)表现出比不掺杂的硫正极材料 (S/CMK-3) 和氮掺杂的硫正极材料 (S/NPC) 更稳定的循 环性能. 这些研究结果证明, 基于化学键合作用的化学 改性碳在改善锂硫电池器件性能, 尤其循环性能方面比 基于物理吸附作用的碳质材料表现出更大的优势. 基于 此, 并考虑到酚醛树脂的原料价格低廉、合成方法高效 简单、丰富的氧结构等特点, 我们设计并以廉价易得的 小分子化合物对苯二酚和甲醛原料, 通过缩聚反应、与 氧化石墨烯原位复合、高温氮化制备了一类新型三维氮 掺杂的碳纳米带固硫载体材料(NCNB 和 NCNB-NG), 通过复合纳米硫进一步得到了碳-硫复合正极材料 $(\mathrm{S} @$
NCNB 和 $\mathrm{S} @ \mathrm{NCNB}-\mathrm{NG})$. 研究发现, NCNB-NG 具有更 高的氮含量、更大的比表面积和更好的导电性，其硫正 极 $\mathrm{S} @ \mathrm{NCNB}-\mathrm{NG}$ 与 $\mathrm{S} @ \mathrm{NCNB}$ 相比, 展现出更优异的 倍率性能和循环稳定性.

\section{2 结果与讨论}

我们利用廉价易得的原料对苯二酚和甲醛在 $180{ }^{\circ} \mathrm{C}$ 水热条件下进行缩聚反应生成酚醛树脂纳米带 聚合物(PHF), 通过原位复合氧化石墨烯得到碳前驱体 (PHF-GO), 接着通过高温氮化掺氮合成氮掺杂的碳纳 米带固硫载体材料(NCNB 和 NCNB-NG), 再通过化学 沉积方法载纳米硫制备 $\mathrm{S} @ \mathrm{NCNB}$ 和 $\mathrm{S} @ \mathrm{NCNB}-\mathrm{NG}$ 正极 材料(图 1).

NCNB-NG 的形貌和微结构通过扫描显微镜和透射 显微镜得到表征. 在图 $2 \mathrm{a}$ 及其内插图以及图 $2 \mathrm{~b}$ 中, 可 以明显观察到碳纳米带 $(\mathrm{NCNB})$ 和石墨烯纳米片 $(\mathrm{NG})$, 并且纳米带和纳米片相互缠绕形成了一种孔尺寸在几 百纳米的三维网络结构. 进一步的高分辨 N 1s X 射线 光电子能谱(XPS)表征结果表明只含有碳和氧的 PHF$\mathrm{GO}$ 复合物前驱体通过高温氮化能够在碳化的同时成功 掺杂氮到石墨烯中和有机物炭化得到的产物中生成 NCNB-NG (图 2c). NCNB-NG 的 N 1s X精细谱可以拟合 成三组峰, 分别对应于吡啶氮(399.1 eV), 吡咯氮(400.0 $\mathrm{eV})$ 和石墨氮 $(401.8 \mathrm{eV})$. 其中吡啶氮占主导, 该类型氮 已被证明可以更有效的起到固硫作用, 提高锂硫电池的 性能 ${ }^{[16]}$. XPS 显示 NCNB-NG 的氮掺杂含量为 $9.7 \%$, 明 显的高于 NCNB 的 $6.5 \%$ 氮掺杂含量(图 S3b)和报道的氮

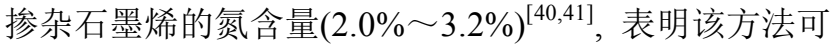
以有效地提高碳骨架的氮含量. 另外, 电导率测试结果 表明, NCNB-NG 具有更高的导电性(表 S1), 而碳载体的 导电性对硫正极的性能发挥至关重要 ${ }^{[16,42]}$. 图 $2 \mathrm{~d}$ 是 NCNB-NG 的氮气吸附-脱附等温线和孔径分布图. 计 算结果表明, NCNB-NG 碳纳米带样品的比表面积为 427 $\mathrm{m}^{2} \cdot \mathrm{g}^{-1}$. 从图 $2 \mathrm{~d}$ 内插图可以看出, 与 $\mathrm{NCNB}$ 具有相似的 孔径分布(图 S3a), NCNB-NG 中主要以介孔和大孔为主, 孔径分布较宽(4 40 nm), 这样的多级孔道结构益于电

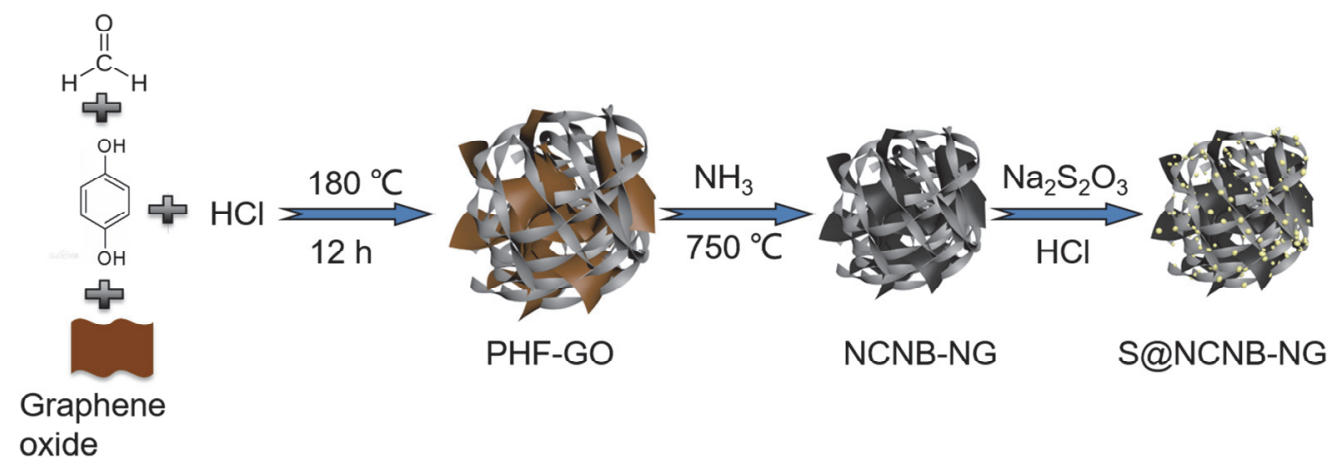

图 1 S@NCNB-NG 正极材料的制备示意图

Figure 1 Schematic illustration of S@NCNB-NG 
(a)

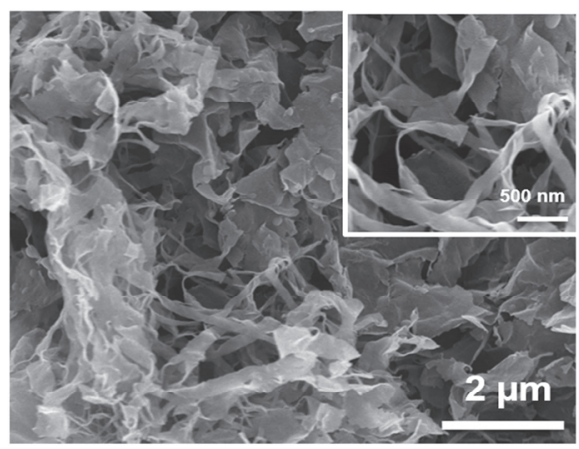

(c)

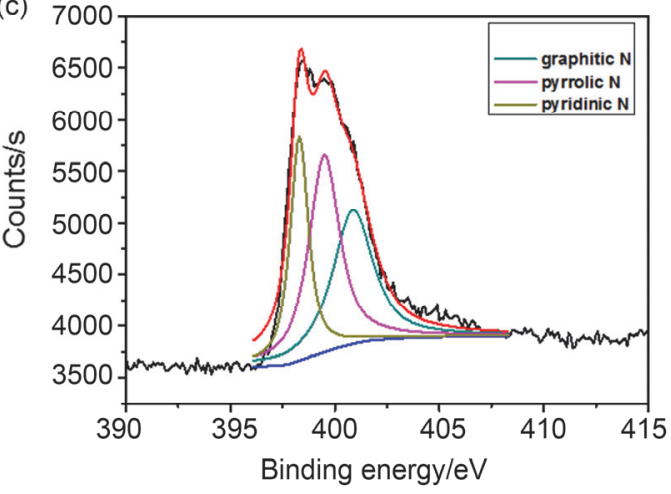

(b)

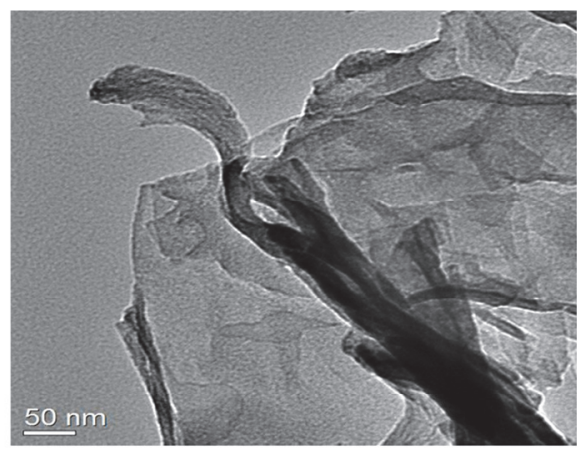

(d)

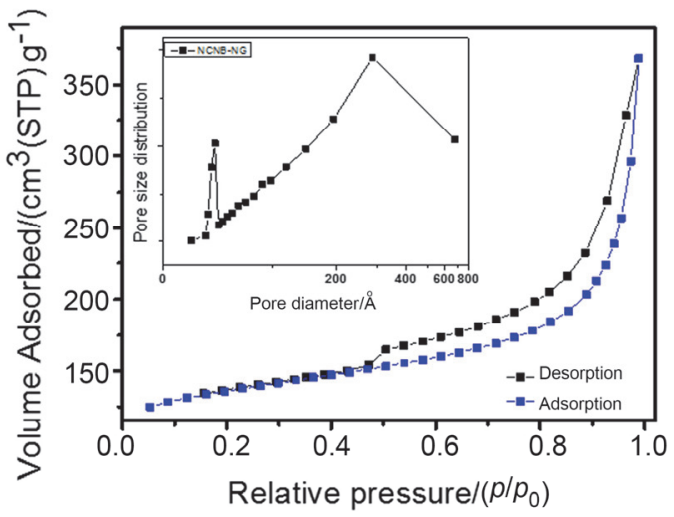

图 2 NCNB-NG 碳载体的扫描电镜图(a), 透射电镜图(b), 高分辨 N 1s XPS 光谱(c)和氮气吸附-脱附等温线和孔分布曲线(d)

Figure 2 (a) SEM images, (b) TEM image, (c) High-resolution N 1s XPS spectrum and (d) Pore diameter and adsorption-desorption curves of NCNB-NG

解液进入孔道, 有利于电解液离子在材料内部的快速传 输, 从而提升锂硫电池的电化学性能 ${ }^{[3,44]}$.

图 3a为 $\mathrm{S} @ \mathrm{NCNB}-\mathrm{NG}$ 正极材料的扫描电镜图. 电 子显微镜扫描照片能够清晰的看出 NCNB-NG 的三维网 络结构, 并且未观察到明显的大硫颗粒, 表明通过化学 沉积方法可以实现 NCNB-NG 载体与纳米硫的有效复 合. 透射电镜照片以及 EDX 硫的元素分析扫描电镜进 一步表明了元素硫是均匀分布在 NCNB-NG 碳骨架上 (图 3b 到图 3f). X 射线衍射图谱结果表明(图 3g), $\mathrm{S} @ N C N B-N G$ 的硫为正交晶相, 与标准卡(JCPDS card no. 24-0733)比较吻合. 热重分析对 S@NCNB-NG 正极 材料进行了表征, 结果表明其硫含量为 53\%(图 3h).

作为对比, 我们用 PHF 和 NCNB 作为硫的载体制 备了 $\mathrm{S} @ \mathrm{PHF}$ 和 $\mathrm{S} @ \mathrm{NCNB}$ 正极材料. 从图 4c、图 4a 和 图 S2 可以看出, 通过高温氮化, NCNB 能够很好地保持 PHF 的三维纳米带网络结构(图 S1a), 带宽为 $50 \sim 100$ $\mathrm{nm}$. 通过类似的化学沉积方法载硫后, 表征结果表明纳 米硫都能均匀地分布在 PHF 和 NCNB 骨架上, 并且发 现正极材料中仍然保留着孔结构, 这更有利于电解液扩 散至活性材料表面(图 4b、图 4d 和图 S1b).

图 5a为 $\mathrm{S} @ N C N B-N G$ 正极组装成锂硫电池后的循 环伏安曲线, 可以明显的观察到硫的特征可逆电化学还 原和氧化峰, 2.23 和 $2.01 \mathrm{~V}$ 阴极峰可归属于硫单质到高 阶硫、低阶硫、硫化锂的还原, $2.47 \mathrm{~V}$ 的阳极峰可归属 于硫化锂到低阶硫、高阶硫、单质硫的氧化 ${ }^{[45,46]}$. 随着
循环的进行, 阳极峰逐渐的向低电势移动, 最后趋于稳 定, 表明经过几圈活化之后, 硫正极的可逆性得到了改 善. 图 $5 \mathrm{~b}$ 和图 $\mathrm{S} 4$ 为 $\mathrm{S} @ \mathrm{NCNB}-\mathrm{NG}$ 正极材料的倍率性 能表征结果. S@NCNB-NG 电极在 $0.05 \mathrm{C}$ 倍率下的初放 容量可以达到 $1338 \mathrm{~mA} \cdot \mathrm{h} \cdot \mathrm{g}^{-1}$. 在 $0.1 \mathrm{C}, 0.2 \mathrm{C}, 0.5 \mathrm{C}, 1$ $\mathrm{C}, 2 \mathrm{C}$ 和 $3 \mathrm{C}$ 倍率下, 可以放出的可逆容量分别为 922 , $720,591,470,374$ 和 $312 \mathrm{~mA} \cdot \mathrm{h} \cdot \mathrm{g}^{-1}$, 当倍率从 $3 \mathrm{C}$ 调回 到 $0.2 \mathrm{C}$, 容量仍然可以恢复到 $631 \mathrm{~mA} \cdot \mathrm{h} \cdot \mathrm{g}^{-1}$. 这些结果 表明该正极材料具有良好的倍率性能. 图 $5 \mathrm{c}$ 为 $\mathrm{S} @ \mathrm{PHF} 、 \mathrm{~S} @ \mathrm{NCNB}$ 和 $\mathrm{S} @ \mathrm{NCNB}-\mathrm{NG}$ 正极材料的电压 容量曲线, S@NCNB-NG 正极材料表现出更高的硫利用 率, 这与其 NCNB-NG 碳载体具有更高的导电性相吻合.

图 5d 和图 $\mathrm{S} 5$ 为 $\mathrm{S} @ \mathrm{PHF}, \mathrm{S} @ \mathrm{NCNB}$ 和 $\mathrm{S} @ \mathrm{NCNB}-$ $\mathrm{NG}$ 正极材料的循环性能测试结果. 在 $0.2 \mathrm{C}$ 倍率下, $\mathrm{S} @ N C N B-N G$ 正极材料在循环 80 圈后的容量为 659 $\mathrm{mA} \cdot h \cdot \mathrm{g}^{-1}$, 而对应的 S@PHF 和 S@NCNB 的容量分别 为 416 和 $503 \mathrm{~mA} \cdot \mathrm{h} \cdot \mathrm{g}^{-1}$. 我们认为 NCNB-NG 的相对高 氮含量和高导电性抑制了电极反应过程中多硫化物向 电解液的扩散并有效改善了硫正极的导电性, 从而使电 池比容量和循环性能得到明显提升. 我们进一步测试了 S@NCNB-NG 正极材料的长循环性能(图 5e). 结果表明 经过两圈的活化后, 仍可以放出 $898 \mathrm{~mA} \cdot \mathrm{h} \cdot \mathrm{g}^{-1}$ 的容量, 循环 100 圈之后, 容量保持在 $612 \mathrm{~mA} \cdot \mathrm{h} \cdot \mathrm{g}^{-1}, 200$ 圈之后, 容量在 $597 \mathrm{~mA} \cdot \mathrm{h} \cdot \mathrm{g}^{-1}, 100$ 圈到 200 圈的每圈衰减率为 $0.025 \%$, 循环 300 圈之后, 容量仍然能保持在 556 

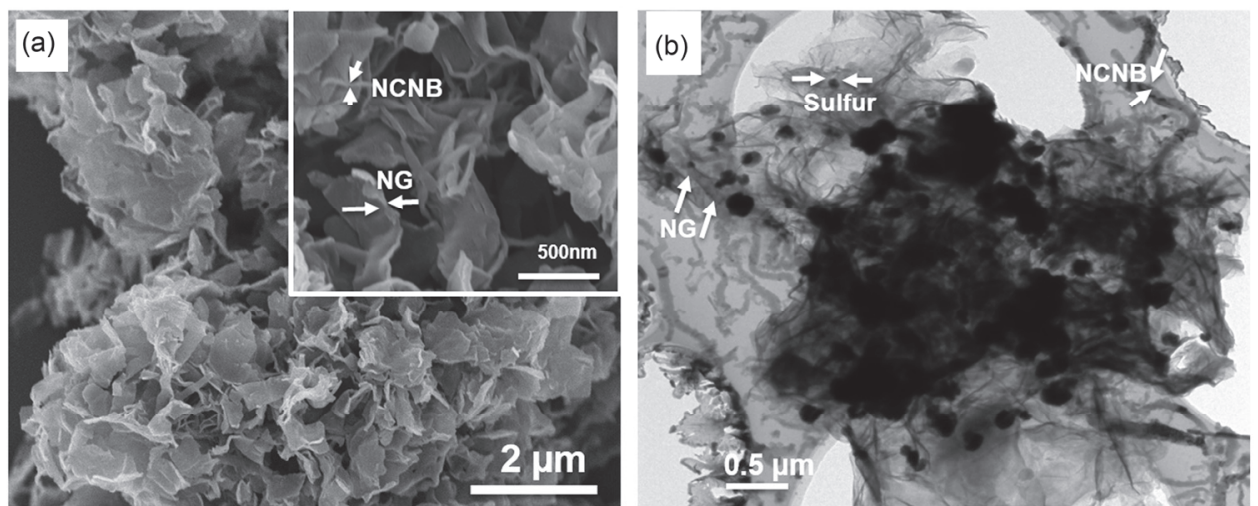

(c)
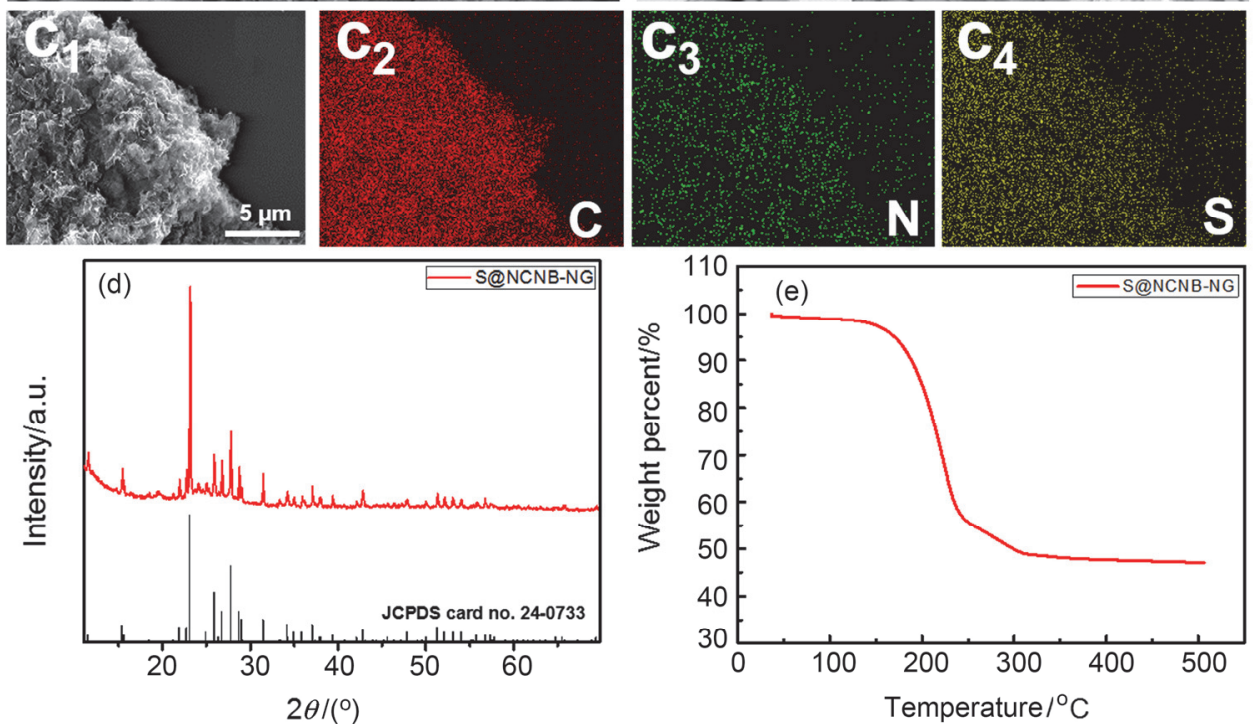

图 3 S@NCNB-NG 正极材料的扫描电镜图(a), 透射电镜图(b), 碳、硫和氮的元素分布图(c), X 射线衍射图谱(JCPDS card no. 24-0733) (d)和热重 图谱(e)

Figure 3 (a) SEM images, (b) TEM image, (c) EDX elemental mapping SEM image for C, S, and $\mathrm{N}$ elements, (d) XRD pattern (JCPDS card no. 24-0733) and (e) TGA curve of S@NCNB-NG
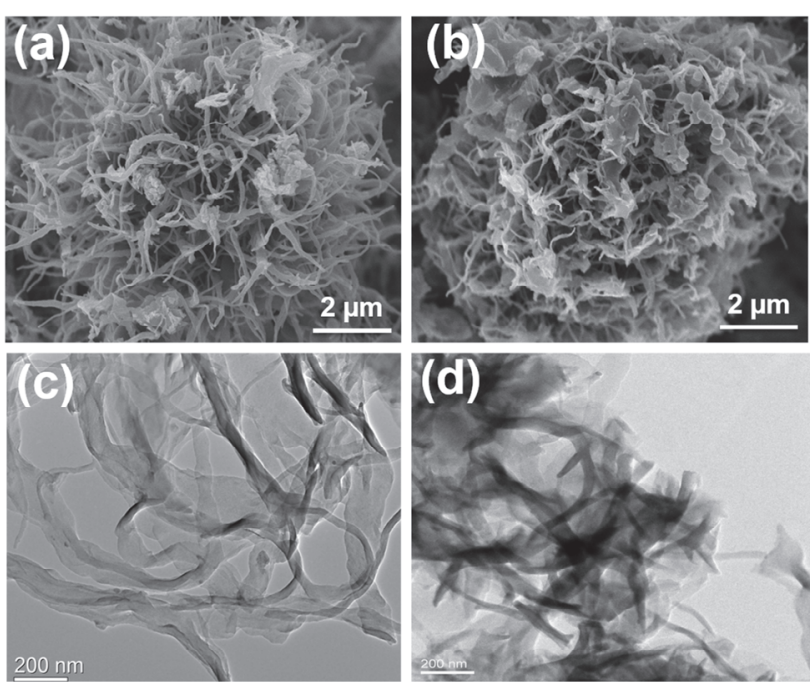

图 4 NCNB 碳载体的扫描电镜图(a), 透射电镜图(c)和 S@NCNB 正 极材料的扫描电镜图(b)和透射电镜图(d)

Figure 4 (a) SEM image of NCNB, (b) SEM image of S@NCNB, (c) TEM image of NCNB and (d) TEM image of S@NCNB

$\mathrm{mA} \cdot \mathrm{h} \bullet \mathrm{g}^{-1}, 100$ 圈到 300 圈的每圈衰减率为 $0.046 \%$, 循
环 300 圈每圈的衰减率为 $0.127 \%$, 库伦效率接近 $100 \%$ (图 S6). 这些结果表明，通过价格低廉、方法简单、易 规模化制备的三维氮掺杂碳纳米带能够有效地抑制锂 硫电池中存在的穿梭效应，并表现出了一定的潜在应用 价值.

\section{3 结论}

综上所述，我们发展了一种简单易行、价格低廉、 易规模化生产新型三维氮掺杂碳纳米带的方法，通过原 位复合氧化石墨烯技术，有效地提高了该类新型材料的 氮含量和导电性，与硫复合制备了锂硫电池的正极材 料, 在 $0.05 \mathrm{C}$ 倍率下, $\mathrm{S} @ \mathrm{NCNB}-\mathrm{NG}$ 电极的初放容量可 以达到 $1338 \mathrm{~mA} \cdot \mathrm{h} \cdot \mathrm{g}^{-1}, 0.2 \mathrm{C}$ 循环 300 圈之后, 容量仍然 能保持在 $556 \mathrm{~mA} \cdot \mathrm{h} \cdot \mathrm{g}^{-1}, 300$ 圈的每圈衰减率为 $0.127 \%$.

\section{4 实验部分}

\section{1 材料制备}

采用改性的 Hummers 法合成氧化石墨烯 

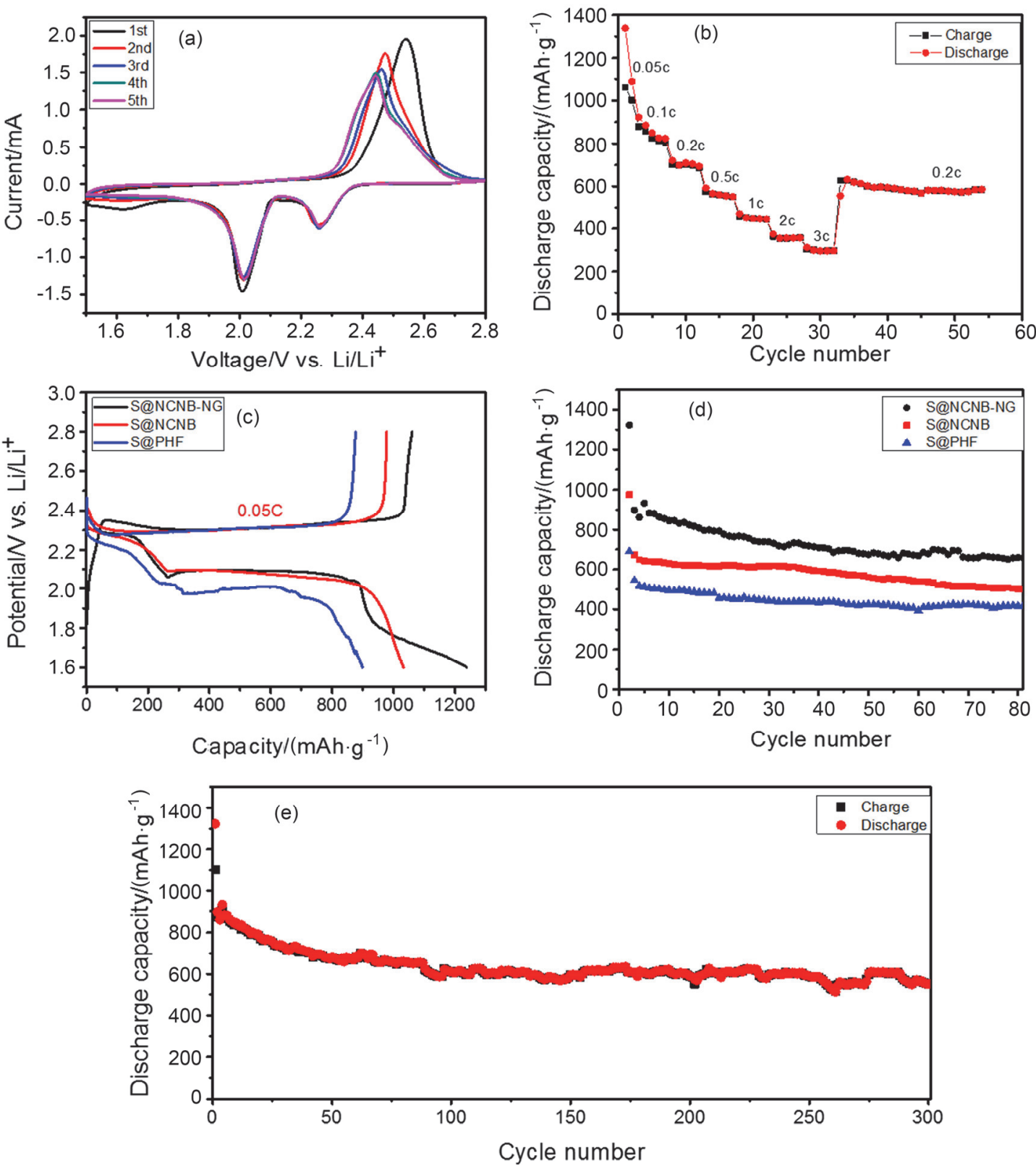

图 $5 \mathrm{~S} @ \mathrm{NCNB}-\mathrm{NG}$ 正极的循环伏安曲线( $\mathrm{a}$ ), 倍率性能(b), $0.05 \mathrm{C}$ 倍率下的 $\mathrm{S} @ \mathrm{PHF}, \mathrm{S} @ \mathrm{NCNB}$ 和 $\mathrm{S} @ \mathrm{NCNB}-\mathrm{NG}$ 正极材料的电压容量曲线( $(\mathrm{c}), 0.2$ C 倍率下 S@PHF, S@NCNB 和 S@NCNB-NG 正极材料的循环性能对比(d), 基于 S@NCNB-NG 正极的锂硫电池的长循环性能(e)

Figure 5 (a) CV profile of the S@NCNB-NG electrode, (b) The rate performance of the S@NCNB-NG electrode, (c) voltage-capacity profiles of the S@PHF, S@NCNB and S@NCNB-NG electrodes at 0.05 C, (d) Cycling performance comparisons between the S@PHF, S@NCNB and S@NCNB-NG electrodes at $0.2 \mathrm{C}$ and (e) Long-term cycling test results of the Li/S cell with S@NCNB-NG electrode

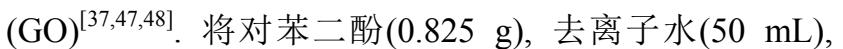
盐酸 $[36 \%$ (质量分数), $15 \mathrm{~mL}], \mathrm{GO}$ 粉末 $(90 \mathrm{mg}$ )和甲醛 [37\%(质量分数), $1.25 \mathrm{~mL}$ 依次放入 $100 \mathrm{~mL}$ 的特氟隆不锈钢高压釜中. 将高压釜密封并在 $180{ }^{\circ} \mathrm{C}$ 的烘箱中 反应 $12 \mathrm{~h}$. 将获得的样品用去离子水洗涤, 并冷冻干燥 $24 \mathrm{~h}$, 得到 PHF-GO 样品. 同样条件下, 不添加 GO 可以 得到 PHF 样品.

将上述得到的 PHF-GO 粉末置于管式炉中在氩气 (99.99\%)流动条件下除掉管中空气和水. 然后将流动的 气体切换成氨气同时调整流速为 $30 \mathrm{sccm}$. 在 $750{ }^{\circ} \mathrm{C}$ 条
件下氮化 $30 \mathrm{~min}$, 随后转换到氩气的条件下冷却至室 温, 获得三维氮掺杂碳纳米带/石墨烯(NCNB-NG). 同 样条件下, 以 PHF 为前驱体, 可以获得 NCNB 样品.

将 NCNB-NG (180 mg)加入到 $180 \mathrm{~mL}$ 的超纯水中, 超声处理 $10 \mathrm{~min}$ 形成稳定的分散液. 然后，以硫代硫酸 钠(1.76 g) 为硫源溶解于 NCNB-NG 分散液中, 在超声 波 ${ }^{[49]}$ 的条件下加入稀盐酸进行原位化学沉积反应. 将 沉淀物过滤、用蒸馏水洗涤三次, 并冷冻干燥 $24 \mathrm{~h}$ 得到 S@NCNB-NG 正极材料. 同样条件下, 以 PHF 和 NCNB 为载体可以获得 $\mathrm{S} @ \mathrm{PHF}$ 和 $\mathrm{S} @ \mathrm{NCNB}$ 正极材料. 


\section{2 材料表征}

采用 SEM (Hitachi S4800)、TEM (Tecnai G2 F20 S-Twin)、XRD (Bruker D8)、ESCALAB 250XI (Thermo Scientific)、TG/DTA 6200 表征材料的形貌、结构和成分. 采用 ASAP 2020 (Micromeritics)测量材料的氮气吸附-脱 附等温线和孔径分布. 采用 Land CT2001 和 Bio-logic VMP3 电化学工作站表征材料的电化学性能.

\section{3 电池的组装及电化学性能表征}

将碳一硫复合正极材料(S@NCNB-NG, S@NCNB composite 和 S@PHF), 碳黑(Super P) 和粘结剂(LA132) 按 $70: 20: 10$ 的质量比制备电极浆料, 涂布, $50{ }^{\circ} \mathrm{C}$ 真 空干燥 $24 \mathrm{~h}$ 得到硫正极. 将隔膜、锂片在氩气气氛手套 箱中与硫正极组装成电池, 电解液为 $1 \mathrm{~mol} / \mathrm{L}$ 二(三氟甲 基磺酰)亚胺锂(LiTFSI)的乙二醇二甲醚与 1,3-二氧戊环 (DME-DOL, 体积比 $1: 1$ ) 溶液 (含 $1 \% \mathrm{LiNO}_{3}$ ). 电池组装 完成后静置 $12 \mathrm{~h}$, 采用蓝电电池测试系统 (Land CT2001)和电化学工作站(Bio-logic VMP-3)进行电化学 性能测试.

\section{References}

[1] Bruce, P. G.; Freunberger, S. A.; Hardwick, L. J.; Tarascon, J. M. Nat. Mater. 2012, 11, 172.

[2] Wang, D. W.; Zhou, G.; Li, F.; Wu, K. H.; Lu, G. Q.; Cheng, H. M.; Gentle, I. R. Phys. Chem. Chem. Phys. 2012, 14, 8703.

[3] Song, M.-K.; Cairns, E. J.; Zhang, Y. G. Nanoscale 2013, 5, 2186.

[4] Larcher, D.; Tarascon, J. M. Nat. Chem. 2015, 7, 19.

[5] Mikhaylik, Y. V.; Akridge, J. R. J. Electrochem. Soc. 2004, 151, A1969.

[6] Diao, Y.; Xie, K.; Hong, X.-B.; Xiong, S.-Z. Acta Chim. Sinica 2013, 71, 508 (in Chinese). ( Э岩, 谢凯, 洪晓斌, 熊仕昭, 化学学 报, 2013, 71, 508.)

[7] Wild, M.; O'Neill, L.; Zhang, T.; Purkayastha, R.; Minton, G.; Marinescub, M.; Offer, G. J. Energy Environ. Sci. 2015, 8, 3477.

[8] Ji, X.; Lee, K. T.; Nazar, L. F. Nat. Mater. 2009, 8, 500.

[9] Liang, C.; Dudney, N. J.; Howe, J. Y. Chem. Mater. 2009, 21, 4724.

[10] Wang, H. L.; Yang, Y.; Liang, Y. Y.; Robinson, J. T.; Li, Y. G.; Jackson, A.; Cui, Y.; Dai, H. J. Nano Lett. 2011, 11, 2644.

[11] Cao, Y. L.; Li, X. L.; Aksay, I. A.; Lemmon, J.; Nie, Z. M.; Yang, Z. G.; Liu, J. Phys. Chem. Chem. Phys. 2011, 13, 7660

[12] Guo, J. C.; Xu, Y. H.; Wang, C. S. Nano Lett. 2011, 11, 4288.

[13] Wei, S. C.; Zhang, H.; Huang, Y. Q.; Wang, W. K.; Xia, Y. Z.; Yu, Z. B. Energy Environ. Sci. 2011, 4, 736.

[14] He, G.; Ji, X.; Nazar, L. Energy Environ. Sci. 2011, 4, 2878.

[15] Song, M. K.; Zhang, Y. G.; Cairns, E. J. Nano Lett. 2013, 13, 5891.

[16] Qiu, Y. C.; Li, W. F.; Zhao, W.; Li, G. Z.; Hou, Y.; Liu, M. N.; Zhou, L. S.; Ye, F. M.; Li, H. F.; Wei, Z. H.; Yang, S. H.; Duan, W. H.; Ye, Y. F.; Guo, J. H.; Zhang, Y. G. Nano Lett. 2014, 14, 4821.
[17] Pope, M. A.; Aksay, I. A. Adv. Energy Mater. 2015, 5, 1500124.

[18] Suo, L. M.; Hu, Y. S.; Li, H.; Armand, M.; Chen, L. Q. Nat. Commun. 2013, 4, 1481 .

[19] Jin, C.-Q.; Xie, K.; Hong, X.-B. Acta Chim. Sinica 2014, 72, 11 (in Chinese). (金朝庆, 谢凯, 洪晓斌, 化学学报, 2014, 72, 11.)

[20] Zhang, S.; Ueno, K.; Dokko, K.; Watanabe, M. Adv. Energy Mater. 2015, 5, 1500117 .

[21] Bauer, I.; Thieme, S.; Brückner, J.; Althues, H.; Kaskel, S. J. Power Sources 2014, 251, 417 .

[22] Chung, S. H.; Manthiram, A. Adv. Mater. 2014, 26, 1360

[23] Huang, J. Q.; Zhang, Q.; Peng, H. J.; Liu, X. Y.; Qian, W. Z.; Wei, F. Energy Environ. Sci. 2014, 7, 347

[24] Zhang, Z. Y.; Lai, Y. Q.; Zhang, Z. A.; Zhang, K.; Li, J. Electrochim. Acta 2014, 129, 55.

[25] Chung, S. H.; Manthiram, A. J. Phys. Chem. Lett. 2014, 5, 1978.

[26] Huang, J. Q.; Zhang, Q.; Wei, F. Energy Storage Mater. 2015, 1, 127.

[27] Huang, J. Q.; Zhuang, T. Z.; Zhang, Q.; Peng, H. J.; Chen, C. M.; Wei, F. ACS Nano 2015, 9, 3002.

[28] Zhang, Y.; Gu, H.; Iijima, S. Appl. Phys. Lett. 1998, 73, 3827.

[29] Zhang, Y.; Iijima, S. Appl. Phys. Lett. 1999, 75, 3087.

[30] Scott, C. D.; Arepalli, S.; Nikolaev, P.; Smalley, R. E. Appl. Phys A-Mater. Sci. \& Proc. 2001, 72, 573.

[31] Zhu, H. W.; Li, X. S.; Jiang, B.; Xu, C. L.; Zhu, Y. F.; Wu, D. H. Chem. Phys. Lett. 2002, 366, 664.

[32] Lange, H.; Sioda, M.; Huczko, A.; Zhu, Y. Q.; Kroto, H. W.; Walton, D. R. M. Carbon 2003, 41,1617.

[33] Guo, J.; Wang, X.; Yao, Y.; Yang, X.; Liu, X.; Xu, B. Mater. Chem. Phys. 2007, 105, 175.

[34] Dong, X. C.; Li, B.; Wei, A.; Cao, X. H.; Chan-Park, M. B.; Zhang, H.; Li, L. J.; Huang, W.; Chen, P. Carbon 2011, 49, 2944.

[35] Choi, H.; Kim, H.; Hwang, S.; Kang, M.; Jung, D. W.; Jeon, M. Scripta Mater. 2011, 64, 601.

[36] Poh, H. L.; Sanek, F.; Ambrosi, A.; Zhao, G.; Sofer, Z.; Pumera, M. Nanoscale 2012, 4, 3515.

[37] Chen, J.; Yao, B. W.; Li, C.; Shi, G. Q. Carbon 2013, 64, 225.

[38] Liu, N.; Lu, Z. D.; Zhao, J.; McDowell, M. T.; Lee, H. W.; Zhao, W. T.; Cui, Y. Nat. Nanotech. 2014, 9, 187.

[39] Yang, C.; Yin, Y.; Ye, H.; Jiang, K.; Zhang, J.; Guo, Y. ACS Appl. Mater. Interfaces 2014, 6, 8789

[40] Qiu, Y.; Zhang, X.; Yang, S. H. Phys. Chem. Chem. Phys. 2011, 13, 12554.

[41] Li, X.; Wang, H.; Robinson, J. T.; Sanchez, H.; Diankov, G.; Dai, H. J. J. Am. Chem. Soc. 2009, 131, 15939.

[42] Huang, J. Q.; Liu, X. F.; Zhang, Q.; Chen, C. M.; Zhao, M. Q.; Zhang, S. M.; Zhu, W. C.; Qian, W. Z.; Wei, F. Nano Energy 2013, 2,314 .

[43] Li, Z.; Jiang, Y.; Yuan, L. X.; Yi, Z. Q.; Wu, C.; Liu, Y.; Strasser, P.; Huang, Y. H. ACS Nano 2014, 8, 9295.

[44] Li, H. F.; Yang, X. W.; Wang, X. M.; Liu, M. N.; Ye, F. M.; Wang, J.; Qiu, Y. C.; Li, W. F.; Zhang, Y. G. Nano Energy 2015, 12, 468.

[45] Ji, X.; Nazar, L. F. J. Mater. Chem. 2010, 20, 9821

[46] Liu, M. N.; Ye, F. M.; Li, W. F.; Li, H. F.; Zhang, Y. G. Nano Res. 2016, 9, 94

[47] Xu, Y. X.; Bai, H.; Lu, G. W.; Li, C.; Shi, G. Q. J. Am. Chem. Soc. 2008, $130,5856$.

[48] Dai, K.; Lu, L. H.; Liu, Q.; Zhu, G. P.; Wei, X. Q.; Bai, J. ; Xuan, L. L.; Wang, H. Dalton Trans. 2014, 43, 6295.

[49] Weng, W.; Pol, V. G.; Amine, K. Adv. Mater. 2013, 25, 1608. 